HOST RESPONSE

\section{Attack is the best form of defence}

The microvilli that make up the brush border of intestinal epithelial cells (IECs) were recently shown to shed intestinal alkaline phosphatase (IAP)-containing vesicles into the gut lumen. Writing in Current Biology, Shifrin et al. now find that these lumenal vesicles provide a mechanism for host innate defences to extend into the gut lumen to attack pathogenic bacteria and their inflammatory products at a distance.

IAP is a host defence factor that can dephosphorylate lipopolysaccharide (LPS) and other bacterial products to reduce their toxicity, and can also limit Toll-like receptor 4 signalling and inflammation. The authors set out to investigate the effect of IAP-enriched lumenal vesicles on the bacterial constituents of the gut lumen. They isolated native vesicles from the small intestine of rats and found that the vesicles dephosphorylated LPS derived from Escherichia coli serotype O55:B5 in a concentration-dependent manner. Incubation with the IAP inhibitor L-phenylalanine blocked vesicle-mediated dephosphorylation of LPS. Vesicle activity was not restricted to E. coli, as LPS from other bacteria, including Pseudomonas aeruginosa and Salmonella enterica subsp. enterica serovar Abortusequi, was also dephosphorylated by the vesicles.

Next, the authors examined material from the rat small intestine using transmission electron microscopy and observed rod-shaped bacteria that were coated in clusters of material resembling small vesicles. Furthermore, confocal microscopy of the same samples using DAPI, DiD and an IAP-specific antibody to stain bacteria, membranes and IAP, respectively, revealed numerous bacteria surrounded by IAP-enriched membranous structures that were likely to be lumenal vesicles.

So what effect do these vesicles have on intestinal bacteria? The authors incubated a confluent monolayer of human IECs with enteropathogenic E. coli (EPEC) str. E2348/69 in the presence or absence of lumenal vesicles. They found that the vesicles substantially reduced EPEC attachment and decreased the pro-inflammatory signalling that would otherwise be stimulated. Interestingly, neither fixing the vesicles in paraformaldehyde nor incubating them with L-phenylalanine prevented them from reducing EPEC attachment, suggesting that this activity is independent of the activity of IAP. In addition to reducing bacterial binding to the IEC surface, lumenal vesicles were found to inhibit bacterial growth, as the addition of vesicles to overnight liquid cultures of EPEC resulted in a decrease in the maximum optical density achieved. Finally, the authors observed that incubation of IECs with EPEC for 6 hours led to an increase in both the total cellular levels of IAP and the amount of lumenal vesicles secreted into the medium.

These findings illuminate a new arm of innate defences in the gut that reaches out into the lumen to preemptively detoxify bacterial products, reduce bacterial growth rates and limit binding of potential pathogens to the IEC surface. It will be interesting to see what other components luminal vesicles contain and use, with the aim of modifying the interactions between microorganisms and the gut.

Andrew Jermy

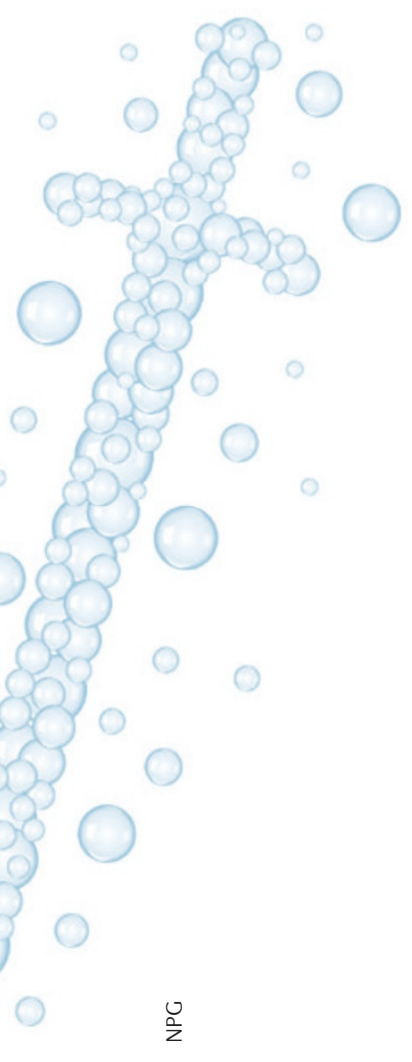

